\title{
Observation of Cooperative Purcell Enhancements in Antenna-Cavity Hybrids
}

\author{
Hugo M. Doeleman, Christian D. Dieleman, Christiaan Mennes, Bruno Ehrler, \\ and A. Femius Koenderink*
}

Cite This: https://dx.doi.org/10.1021/acsnano.0c05233

Read Online

ACCESS | 네 Metrics \& More | 回 Article Recommendations | st Supporting Information

ABSTRACT: Localizing light to nanoscale volumes through nanoscale resonators that are low loss and precisely tailored in spectrum to properties of matter is crucial for classical and quantum light sources, cavity QED, molecular spectroscopy, and many other applications. To date, two opposite strategies have been identified: to use either plasmonics with deep subwavelength confinement yet high loss and very poor spectral control or instead microcavities with exquisite quality factors yet poor confinement. In this work we realize hybrid plasmonic-photonic resonators that enhance the emission of single quantum dots, profiting from both plasmonic confinement and microcavity quality factors. Our experiments directly demonstrate how cavity and antenna jointly realize large cooperative Purcell enhancements through interferences. These can be controlled to engineer arbitrary Fano lineshapes in the local density of optical states.
$\mathrm{R}$ eaching extremes of light confinement in space and time is a decade-long quest in photonics, as it unlocks the strong light-matter interaction regime that underlies single-photon sources and cavity QED, ${ }^{1-3}$ nanolasers, ${ }^{4,5}$ single-molecule sensitive sensors and spectroscopies, $^{6-8}$ and cavity optomechanics. ${ }^{9}$ Many of these applications hinge on the ratio of the resonator quality factor $Q$ and the confinement volume $V$ through the dimensionless Purcell factor $F=3 / 4 \pi^{2} \lambda^{3} \mathrm{Q} / V$. While light-matter interaction scales with $Q / V$, applications generally require independent control of $Q$ without compromising $Q / V$, since one typically needs to match resonator line widths to the spectral bandwidth of an emitter. Unfortunately, realized structures usually excel either exclusively in spatial confinement at the cost of $Q$ or vice versa. On one hand, plasmonic hot spots provide ultratight confinement to volumes below $\lambda^{3} / 10^{5},{ }^{7,10,11}$ yet only for $Q$ $\approx 10$ optical cycles. Conversely, dielectric microcavities store light for up to $10^{5}-10^{9}$ optical cycles, yet generally the associated modes are spread over a volume $V \geq \lambda^{3}$, 2,6,9 with the exception of recent slotted-nanobeam-based designs. ${ }^{12}$ There is hence a strong need for a constructive route toward resonators that leverage plasmonic antennas for ultratight confinement, yet operate at any desirable $Q$. A few recent reports suggest that plasmonic nanoantennas with photonic microcavities can realize this target. ${ }^{13-16}$ However, the path to success is narrow, as the most obvious designs, e.g., having both antenna and cavity resonant with each other, actually lead to fully destructive interference in Purcell factor. ${ }^{17,18}$ Instead, one must precisely control coherent effects whereby antenna and microcavity cooperatively boost the Purcell factor or local density of optical states (LDOS). According to recent theory, appropriate detunings might optimize LDOS to far exceed that of the plasmonic antenna alone, while bandwidth can be chosen over orders of magnitude. ${ }^{18,19}$ While antenna-cavity hybrid systems have been experimentally studied, ${ }^{16,20-25}$ these crucial advantages for strong light-matter interaction, i.e., extreme line width tunability and strong LDOS boosts, have not been observed.

In this Article we realize hybrid plasmonic-photonic resonators and report LDOS measurements on single-photon emitters precisely placed in their plasmonic hotspot. The hybrid resonators are based on high- $Q$ micron-sized $\mathrm{Si}_{3} \mathrm{~N}_{4}$

Received: June 24, 2020

Accepted: September 1, 2020

Published: September 1, 2020 
microdisk cavities and aluminum antennas, coupled to single quantum emitters (see Figure $1(\mathrm{a}-\mathrm{c})$ ). The system directly
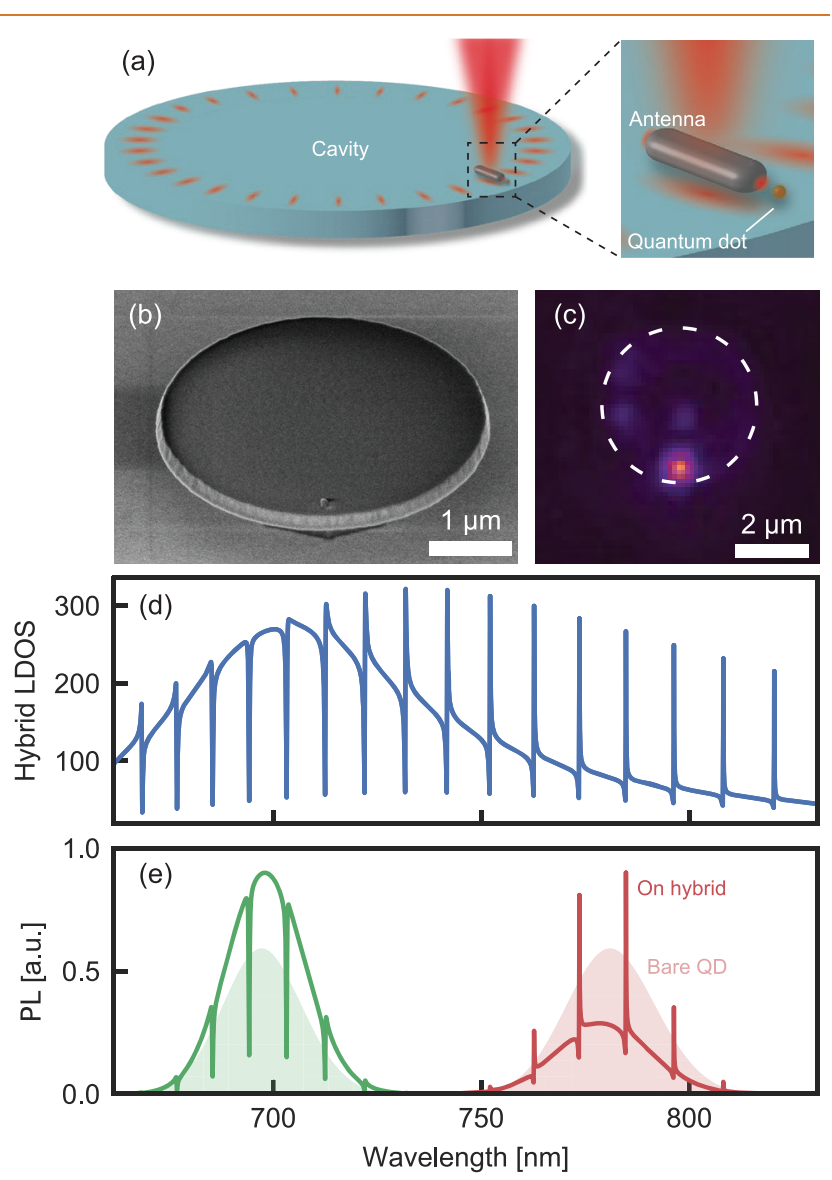

Figure 1. Fluorescence in a cavity-antenna hybrid. (a) Cartoon of our hybrid system, with a nanoantenna placed on a microdisk cavity. A quantum dot at the tip of the antenna couples to the hybridized mode. (b) SEM image of a hybrid studied in this work. (c) Fluorescence image from an example hybrid system, homogeneously pumped. The bright spot at the antenna location signals emission from locally deposited quantum dots. Disk outline is indicated by the dashed circle. (d) Example of a calculated hybrid LDOS spectrum. A broad antenna resonance couples to narrow cavity resonances, leading to Fano lineshapes. (e) Examples of calculated quantum dot emission spectra at resonance (green line) or red-detuned (red line) from the antenna resonance. Shaded areas indicate bare QD spectra. A quantum dot coupled to the hybrid system has the spectral shape of its emission modified in proportion to LDOS (if collection efficiency is constant).

demonstrates Fano-lineshape engineering of $\operatorname{LDOS}^{26}$ and supports hybrid modes with highly tunable quality factors of $>10^{3}$ and mode volumes in between values for the cavity and antenna. Figure 1(d) shows an example calculation of the LDOS spectrum for such a system as would be felt by a quantum emitter right at the antenna. ${ }^{18}$ The broad resonance of the bare antenna LDOS is punctuated by a comb of narrow features, each corresponding to a whispering-gallery mode. These lines have the form of transparency dips for cavity modes on resonance with the antenna, yet LDOS maxima for red-detuning. This calculation indicates that on resonance the cavity spoils the antenna LDOS, while for red-detuning, instead, cavity and antenna cooperatively enhance LDOS. In fact, the LDOS enhancement can exceed that of the antenna, but without the usual plasmonic limitation on $Q$. In this work we show by tapered-fiber spectroscopy how to precisely control the hybrid mode $Q$ over orders of magnitude by detuning and antenna-cavity coupling strength. Next, we interrogate the mode volume with broadband quantum emitters. Their photoluminescence spectra (sketch in Figure $1(\mathrm{e})$ ) are expected to directly reveal the predicted Fano features in LDOS.

\section{RESULTS AND DISCUSSION}

Tuning Hybrid Mode Line Widths. The salient promise of cavity-antenna hybrids is that they provide plasmonic mode confinement and Purcell factors, ${ }^{18}$ yet with line widths that can be freely chosen over orders of magnitude and up to that of the microcavity. Our aim is to quantify hybridized mode properties as a function of the bare cavity $Q$ and mode volume, and the bare antenna resonance. We fabricated hybrids consisting of silicon nitride $\left(\mathrm{Si}_{3} \mathrm{~N}_{4}\right)$ whispering-gallery-mode microdisk cavities coupled to single aluminum rod antennas $(\sim 50 \mathrm{~nm}$ width, $40 \mathrm{~nm}$ height, and length between 68 and $188 \mathrm{~nm}$ ) with two-step electron-beam lithography (Figure 2(a,b)). We pair cavities of 15,12 , and $8 \mu \mathrm{m}$ diameter with 10 different antenna lengths between 68 and $188 \mathrm{~nm}$. By varying cavity diameter and analyzing both the fundamental and next higher $\left(m_{r}=0\right.$ respectively 1) radial order mode, we gain access to a large range of cavity mode volumes. At the same time, the chosen antenna lengths sweep the antenna resonance through the cavity resonance.

We analyze the hybrid mode structure by cavity spectroscopy using a tapered fiber ${ }^{27,28}$ (see Figure 2c) as input/output channel and a narrowband tunable diode laser $(765-781 \mathrm{~nm}$ wavelength) as driving. We monitor fiber reflection and transmission while simultaneously collecting scattered light by a high-NA objective. An exemplary broadband spectrum is shown in Figure $2 \mathrm{~d}$, showing multiple resonances of different $m_{r}$. Typical scattering, reflection, and transmission spectra near a hybrid resonance are shown in Figure $2(\mathrm{~g}-\mathrm{i})$. The antenna splits the intrinsically degenerate clockwise and anticlockwise cavity modes into standing waves. The mode with its node at the antenna is unperturbed, as it is uncoupled from the antenna. In contrast, the hybrid mode with its antinode at the antenna is shifted and broadened as a result of hybridization with the antenna plasmon mode. This identification is verified by the scattering images. Figure $2 \mathrm{e}$ and $\mathrm{f}$ show that at the broad mode the antenna is far brighter than the rest of the disk, indicating hybridization of disk and antenna. The unperturbed mode, in contrast, shows uniform scattering from the disk edge.

We extract resonance frequencies $\omega_{\mathrm{c}}$ and line widths $\kappa$ from simultaneous fitting of transmission, reflection, and scattering spectra (red dashed line in Figure 2(g-i); see Supporting Information for derivation). For each hybrid resonance the adjacent unperturbed mode serves as a yardstick to measure the hybrid frequency shift and broadening against. The unperturbed cavity line widths lie between 1 and $4 \mathrm{GHz}$ (bare cavity $\left.Q \approx(1-4) \times 10^{5}\right)$, while resonance frequencies are reproducible to within $0.04 \%$ ( $2 \%$ of the cavity-free spectral range). This translates to a reproducibility in disk diameter of $2-3 \mathrm{~nm}$. Figure $2(\mathrm{j}, \mathrm{k})$ show the line width difference $(\mathrm{j})$ and frequency shift $(\mathrm{k})$ of the hybrid $m_{r}=0$ mode with respect to the unperturbed mode, as a function of antenna length. Both $m_{r}=0$ and $m_{r}=1$ modes (see Supporting Information) in all disk families show increasing broadening with increasing 


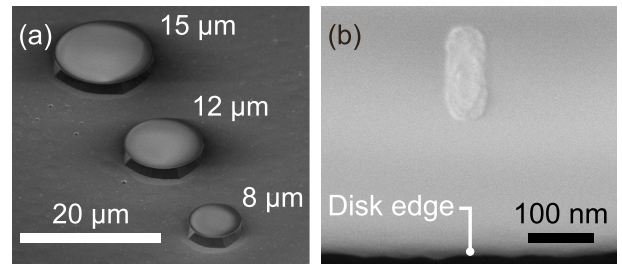

(c)
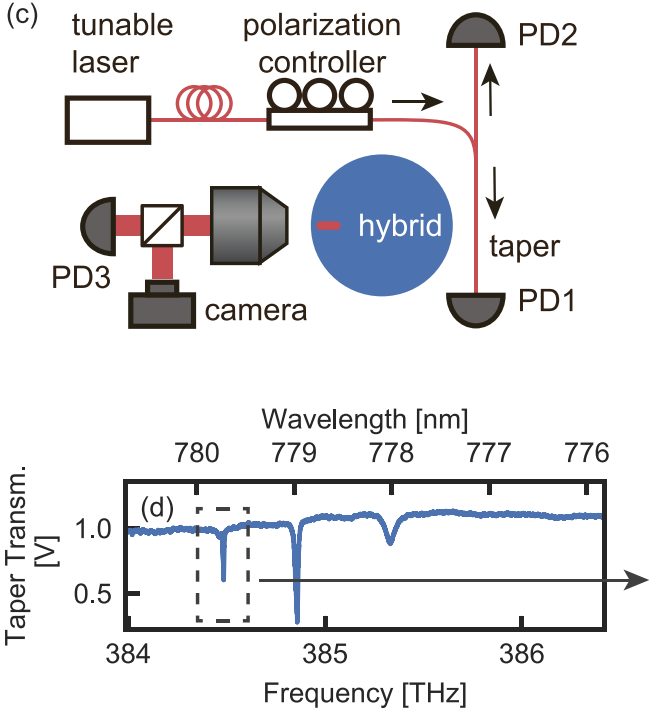
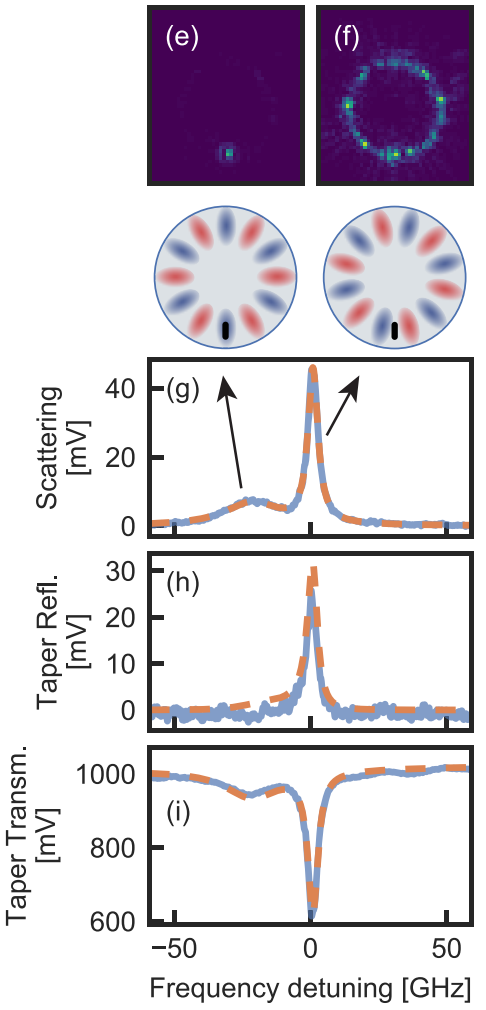
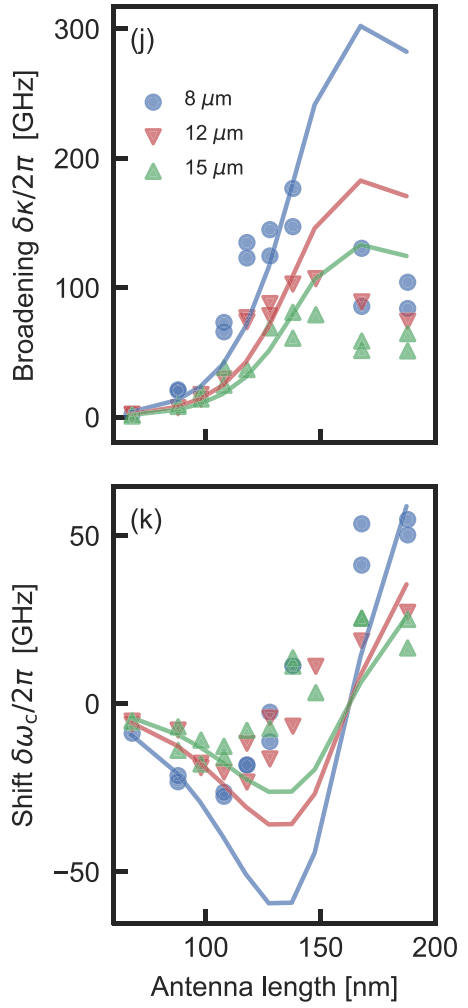

Figure 2. Bandwidth tuning in hybrid systems. (a) Scanning electron micrograph (SEM) of cavity-antenna hybrids, with three disk sizes. The disks are supported by a silicon pedestal. (b) SEM zoomed-in on an Al antenna close to the disk edge. (c) Tapered-fiber spectroscopy setup. The cavity is excited through the taper, and transmission, reflection, and scattering are monitored on photodiodes PD1, PD2, and PD3, respectively. (d) Broadband transmission spectrum measured for a hybrid with $8 \mu \mathrm{m}$ disk diameter and 88 nm antenna length, showing three dips corresponding (from left to right) to the $m_{r}=0,1,2$ modes, respectively. (e, f) Camera images of the hybrid at the frequencies of the perturbed (e) and unperturbed (f) modes. The taper was located at the top edge of the disk, and the antenna at the bottom. Each image is normalized to its maximum. $(\mathrm{g}-\mathrm{i})$ Narrowband scattering $(\mathrm{g})$, reflection $(\mathrm{h})$, and transmission $(\mathrm{i})$ spectra near the fundamental $\left(m_{r}=0\right)$ WGM in the same hybrid as shown in (d). The broad and narrow resonances correspond respectively to the perturbed and unperturbed modes, with mode profiles sketched (not to scale) in the cartoon above (g). Data (blue) are fitted (orange dashes) with a global fit to all three spectra. $(j, k)$ Line width difference $(j)$ and frequency shift $(k)$ of the perturbed $m_{r}=0$ mode, with respect to the unperturbed mode, as a function of antenna length. Markers show experimental data, and the lines represent predictions from perturbation theory. Each marker corresponds to a different physical structure.

antenna length, up to a length around $150 \mathrm{~nm}$, after which the line width drops again. The shift, on the other hand, is dispersive: modes are red-shifted for small antennas and blueshifted for larger antennas. The line width can be chosen at will over a range of two orders of magnitude by changing the antenna length, e.g., from $\sim 1$ to $\sim 100 \mathrm{GHz}$ for the fundamental mode in $12 \mu \mathrm{m}$ disks. Even the most strongly coupled hybrids have high $Q$ exceeding $10^{3}$.

The physics of Figure $2(\mathrm{j}, \mathrm{k})$ is explained by BetheSchwinger cavity perturbation theory, ${ }^{29}$ which states that the change $\delta \widetilde{\omega}_{\mathrm{c}}=\delta \omega_{\mathrm{c}}-i \delta \kappa / 2$ in complex resonance frequency of a cavity by a localized perturbation of optical polarizability $\alpha\left(\omega_{\mathrm{c}}\right)$ is given as

$$
\frac{\delta \widetilde{\omega}_{\mathrm{c}}}{\omega_{\mathrm{c}}}=-\frac{1}{2 \epsilon_{0} \epsilon V_{\mathrm{eff}}} \alpha\left(\omega_{\mathrm{c}}\right)
$$

with $\omega_{\mathrm{c}}$ the original cavity resonance frequency, $V_{\text {eff }}$ its effective cavity mode volume, $\epsilon_{0}$ the vacuum permittivity, and $\epsilon$ the relative permittivity of the medium at (but in absence of) the perturbation. This model predicts that cavity broadening $\delta \kappa$ should trace $\mathcal{J}[\alpha]$, which peaks at antenna resonance. The frequency shift $\delta \omega$ should be dispersive exactly as we observe, tracing $\mathcal{R}[\alpha]$. To go beyond qualitative comparison, we determined $V_{\text {eff }}$ and $\alpha$ from full-wave simulations of the separate disks and antennas. The resulting perturbation theory predictions (solid lines in Figure $2(\mathrm{j}, \mathrm{k})$ ) are fully determined from simulations, without any adjustable parameter.

For all disk sizes (mode volumes) we observe good agreement between theory and data especially for the broadening, up to antennas of $140 \mathrm{~nm}$ length. This agreement evidences both the role of polarizability and the fact that perturbation is inversely proportional to the cavity mode volume. For very long antennas broadenings are overestimated by perturbation theory, which results either from differences in optical constants of $\mathrm{Al}$ between experiment and simulations or from retardation effects not accounted for by BetheSchwinger theory. Regarding the frequency shifts (Figure $2 \mathrm{k}$ ), we find qualitative agreement with theory, with red-shifts transitioning to blue-shifts. Quantitative agreement is likely hampered by the same difference in optical constants and the fact that disk roughness can cause shifts of similar magnitude, yet at negligible broadening, as compared to the antenna. ${ }^{30}$ Nonetheless, data and theory correspond very well, considering that the comparison is in absolute units of frequency with no adjustable parameter. Reported perturbation experiments generally tested only proportionality of frequency shifts to the 

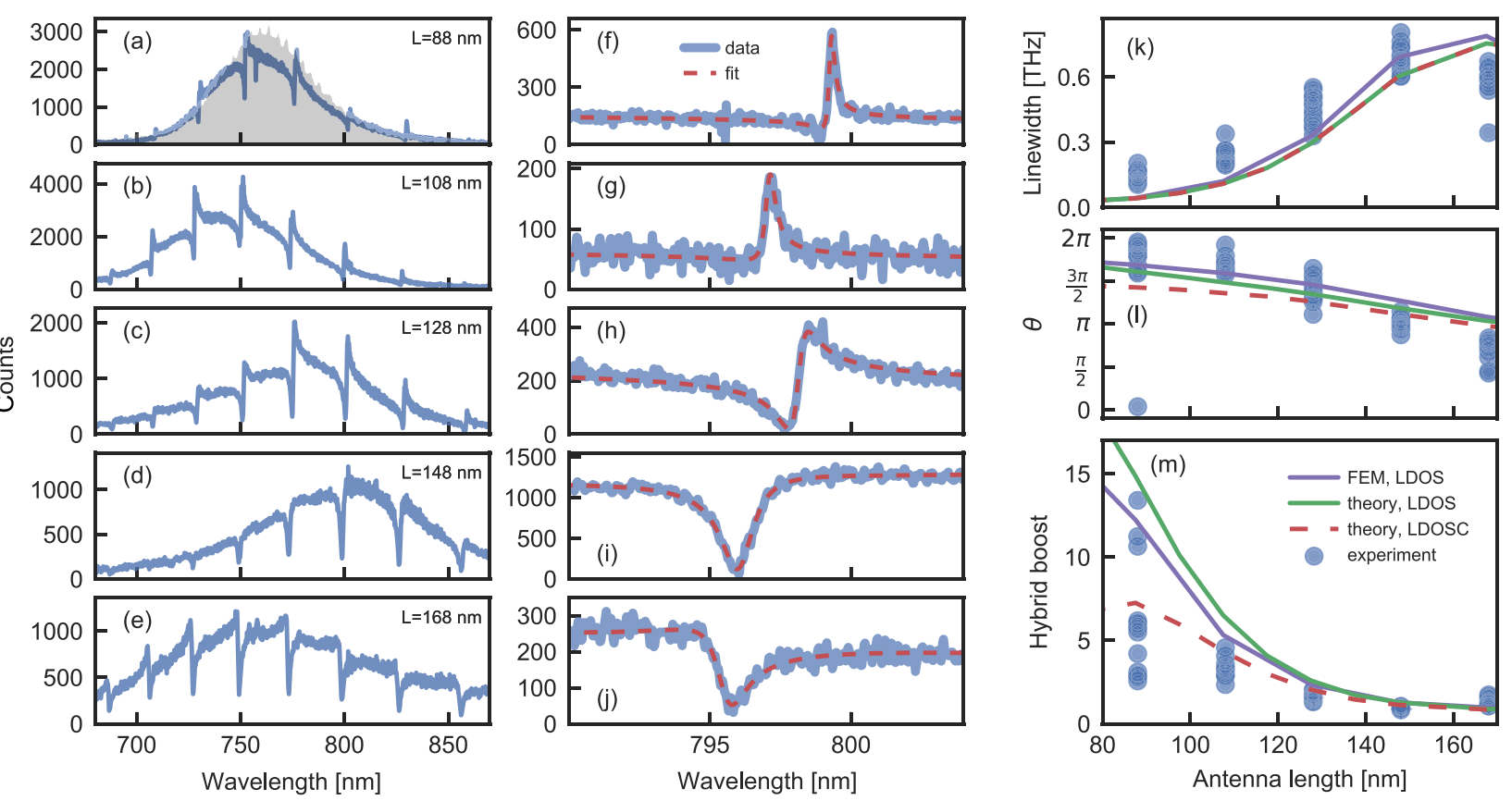

Figure 3. Fluorescent emission spectra in hybrid systems. (a-e) Broadband emission spectra from quantum dots on hybrids with equal disk size and five different antenna lengths $L$ (indicated). The dispersive Fano resonances change gradually from a peak to a dip as $L$ increases. The shaded region in (a) shows the spectrum of a single quantum dot on a flat $\mathrm{Si}_{3} \mathrm{~N}_{4}$ surface. (f-j) High-resolution emission spectra, zoomed-in on the mode near $800 \mathrm{~nm}$ wavelength, for the same hybrids as in (a)-(e). We show data (blue) and Fano fit (red). (k) Fano resonance line width, showing a strong increase as the antenna is tuned to resonance (around $150 \mathrm{~nm}$ length). (1) Fano phase $\theta$ transitions from a peak $(\theta \approx 0)$ for short antennas to a $\operatorname{dip}(\theta \approx \pi)$ for antennas at resonance. For the largest antennas, the resonance takes on a Fano shape with opposite asymmetry, i.e., $\theta<\pi$. $(\mathrm{m})$ Hybrid boost factor is strongest for small antennas, where cavity modes are far from antenna resonance. All experimental data (blue markers) in panels $(k)-(m)$ are obtained from fits as shown in panels $(\mathbf{f})-(j)$. Results are compared to LDOS (green line) or collected LDOS (LDOSC, red dashed line) as predicted by coupled-oscillator theory and to LDOS obtained from finite element (FEM) simulations on the full hybrid system (purple line).

local cavity mode strength by scanning sharp probes of uncalibrated polarizability through the mode of a single cavity. ${ }^{31-34}$ This work constitutes a quantitative, systematic comparison of perturbation to the ratio of polarizability and mode volume across many distinct devices. Key to this comparison is that fabrication of the hybrids is exceptionally robust.

Fluorescence Control. Having established that antennacavity systems show sharp hybrid modes, we assess the potential for light-matter interaction by plasmonic confinement. This is quantified by LDOS, which cannot be obtained from cavity spectroscopy but requires interrogation with fluorescent emitters. We employ a third lithography step to place $\mathrm{CdSeTe} / \mathrm{ZnS}$ core/shell quantum dots (Invitrogen Qdot 800 ITK Organic) at the tips of the aluminum antennas with high selectivity and success rate (see Supporting Information). The technique is adapted from earlier reports ${ }^{35,36}$ and relies on linker chemistry to bind quantum dots (QDs) selectively to aluminum oxide, in combination with a resist mask for localization to within $100 \mathrm{~nm}$. We first examine hybrids with multiple quantum dots. Figure 1c shows a fluorescence image demonstrating the selective quantum dot placement. We analyze hybrids with five disk diameters between 3960 and $4120 \mathrm{~nm}$. In these small disks all higher-order radial modes are suppressed, cavity mode volumes are reduced, and the larger hybrid line widths are well matched to the spectrometer resolution. Each disk contains one antenna placed $300 \mathrm{~nm}$ from the disk edge (Figure 1b), and we test five antenna lengths between 88 and $168 \mathrm{~nm}$ to tune the antenna through the cavity spectrum. We pump at $640 \mathrm{~nm}$ wavelength and detect fluorescence from an area on the sample of $\sim 1 \mu \mathrm{m}$ diameter using a grating spectrometer with $\sim 0.4 \mathrm{~nm}$ spectral resolution for broadband spectra and $\sim 0.1 \mathrm{~nm}$ for highresolution spectra, respectively.

Figure 3a shows a broadband fluorescence spectrum from quantum dots at the antenna apex in a hybrid system. The broad intrinsic QD ensemble emission spectrum is strongly modulated by sharp asymmetric Fano-type resonances, with a spacing set by the cavity-free spectral range. Note that, due to the placement of the QDs at the antenna tip, they cannot emit into the "unperturbed" cavity modes, which have zero field strength at the QD location. The observed Fano lineshapes are strongly reminiscent of the asymmetric LDOS resonances predicted for hybrid photonic-plasmonic resonators ${ }^{17,18}$ and sketched in Figure 1d. The notion that each Fano lineshape originates from an hybrid LDOS resonance is supported by the fact that they only appear when both pump and detection polarization are along the antenna long axis (see Supporting Information). If this notion is indeed true, the lineshapes should depend strongly on cavity-antenna detuning. ${ }^{18}$ Figure $3(\mathrm{a}-\mathrm{e})$ show broadband spectra for hybrids with five different antenna lengths and consequently different cavity-antenna detunings. The Fano resonances gradually evolve from peaked for short antennas (i.e., cavity far-red-detuned) to complete destructive interference for antenna lengths of $148 \mathrm{~nm}$, to Fano peaks of opposite asymmetry for long antennas. This change of Fano asymmetry is even more clearly evident in highresolution spectra (Figure $3(f-j)$ ). The Fano lines imply that emission enhancement results from a coherent interplay of two 

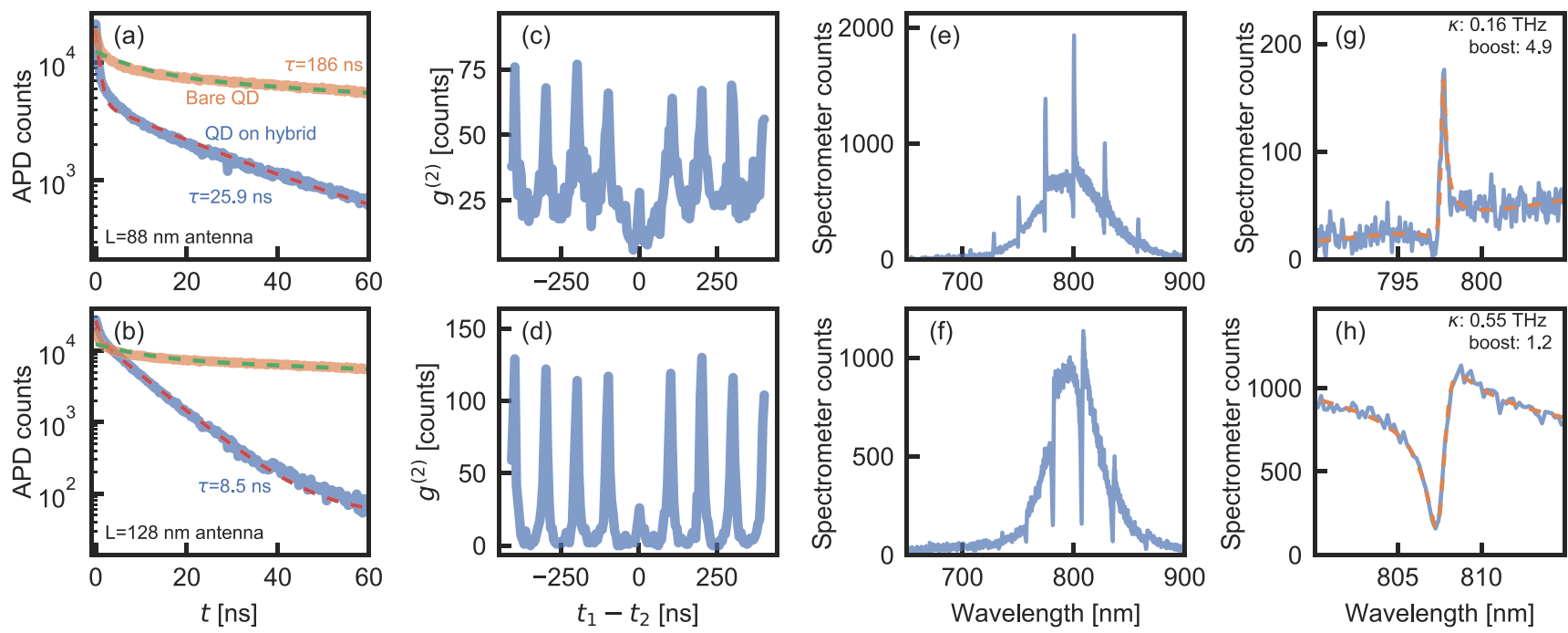

Figure 4. Single quantum dots on hybrids. Single quantum dot excited state decay (a, b), second-order correlation function $g^{(2)}$ (c, d), and broadband $(e, f)$ and narrowband $(g, h)$ spectra. We show data for two different quantum dots on hybrids with antenna lengths $L$ of $88 \mathrm{~nm}$ $(a, c, e, g)$ and $128 \mathrm{~nm}(\mathrm{~b}, \mathrm{~d}, \mathrm{f}, \mathrm{h})$. Decay curves of an example "bare" quantum dot on SiN are also shown in (a) and (b). Dashed lines are fits to the data. As the $88 \mathrm{~nm}$ antenna is far off-resonant with the quantum dot, it shows a moderate lifetime decrease, yet a strong hybrid boost. The $128 \mathrm{~nm}$ antenna is near-resonant, showing a strong lifetime decrease and a moderate boost. Note that decay in (a) and (b) is not perfectly single-exponential due to small, fast-decaying background contributions, which however contribute $<10 \%$ of the total signal.

coupled resonances. The antenna detuning determines whether LDOS shows a transparency line or enhancement.

To quantify the Fano lineshapes, we fit the high-resolution spectra with $I(\omega)=\left|E_{2} \mathrm{e}^{i \theta}+E_{1} \frac{\kappa / 2}{-i \Delta+\kappa / 2}\right|^{2}$, with $\Delta$ and $\kappa$ frequency detuning and line width and $\theta$ the Fano phase. ${ }^{37}$ Example fits are shown in Figure $3(\mathrm{f}-\mathrm{j})$. Assembling statistics from all disk diameters and fitting the different modes in each hybrid between 720 and $800 \mathrm{~nm}$ wavelength evidence that the line width of the hybrid emission signature depends strongly on antenna length (Figure $3(\mathrm{k})$ ). As for the taper spectroscopy, the hybrid line width strongly increases up to antenna lengths of $148 \mathrm{~nm}$, where cavity and antenna are on resonance. A plot of Fano phase $\theta$ in Figure 3(1) furthermore confirms that the behavior in Figure $3(\mathrm{a}-\mathrm{j})$ is generic: hybrid resonances change from nearly Lorentzian $(\theta \approx 0)$ for short antennas, to transparency dips $(\theta \approx \pi)$ at antenna resonance, and to opposite asymmetry $(\theta<\pi)$ at $168 \mathrm{~nm}$ antenna length. Finally, the Fano fit gives access to the strength of the resonances compared to the background at the same frequency. We call this ratio "hybrid emission boost". Figure $3(\mathrm{~m})$ shows that for antennas near resonance $(140 \mathrm{~nm})$ hybrid emission boosts are small. Instead, for short antennas, peak heights can be up to 14 times over the background. This hybrid emission boost factor should be understood as a 14-fold LDOS boost over the enhancement provided by the antenna alone.

The emission enhancement spectra appear to directly follow the predicted LDOS for hybrid systems (Figure 1(d)). In this interpretation, the hybrid boost reports on the Purcell factor of the hybrid mode relative to the "background" LDOS provided by the bare antenna at that frequency. At first sight, this viewpoint conflicts with the rule of thumb that measured emission intensities do not quantify LDOS. The basis for this rule is that two-level systems of unit quantum efficiency emit exactly one photon per absorbed photon irrespective of fluorescence lifetime enhancement, i.e., irrespective of LDOS. However, our quantum dots are not two-level emitters but have a $\sim 50 \mathrm{~nm}$ spectral width, far exceeding the natural line width. The LDOS spectrum directly modifies the branching ratio between all possible transitions that an emitter can undergo. $^{38-42}$ Since emission frequency directly encodes which transition occurred, the emitter spectrum directly reveals the spectrum of LDOS variations (see Supporting Information).

Our argument that Fano features in spectra report on spectral LDOS variations (normalized to average LDOS over the emitter spectrum) is supported by both a coupled oscillator model and full-wave simulations to obtain LDOS (see Supporting Information). Since a pitfall of intensity measurements is that they depend on emission collection efficiency, we include collection efficiency in our coupled oscillator model and extract the "collected part of the LDOS", or LDOSC. The modeling (curves in Figure $3(\mathrm{k}-\mathrm{m})$ ) quantitatively matches the data in terms of Fano line width, asymmetry, and hybrid boost. Since cavity leakage is poorly collected by our objective, collection efficiency at resonance with the hybrid mode is always smaller than off-resonance (i.e., for the bare antenna mode). Hence, the hybrid boost extracted from experiment is a lower bound to the actual hybrid LDOS variation. We therefore anticipate that the hybrid LDOS in our family of hybrids, normalized to free space, exceeds the product of the measured hybrid boost $(>14 \times)$ and the bare plasmon-antenna LDOS enhancement. Bare antenna enhancements can be on the order of 10-100 for the antennas in this work. Even if absolute LDOS varies significantly with the emitter-antenna distance, the hybrid boost that comes on top of the antenna LDOS is not strongly distance dependent (see Supporting Information) and is hence a robust experimental metric.

Measuring LDOS Using Single Quantum Dots on Hybrids. Finally, we demonstrate hybrid photonic-plasmonic resonators driven by single quantum emitters, created by fabricating devices with few quantum dots, and intentional bleaching of excess quantum dots until a single one was left. These samples were measured in the same setup as used for 
the fluorescence spectra, yet here we vary between sending the collected fluorescence to a spectrometer or to a pair of singlephoton detectors in a Hanbury-Brown-Twiss configuration. By correlating the photon arrival times to the pump pulses or cross-correlating the arrival times on both detectors, the fluorescence lifetime $\tau$ or the second-order correlation function $g^{(2)}$ can be obtained. The latter can evidence the presence of a single photon emitter through photon antibunching, i.e., a dip at zero time delay by more than 50\%. We identified 16 structures with just a single quantum dot, as evident from intermittency and photon antibunching. Figure 4 shows fluorescence decay traces, second-order correlation functions $g^{(2)}$, and broadband and narrowband spectra for two single quantum dots. For antibunching and fluorescence lifetime traces we postselected photon counting data for all the time intervals where the quantum dot was in the bright state, to suppress background from the silicon nitride. As even the single quantum dots have a broad spectrum, we again observe Fano lines. Conversely, since fluorescence lifetime is a metric that integrates over all possible transitions, we expect that it will report just the spectrally averaged LDOS. Thus, lifetime will essentially report just the LDOS change induced by the antenna, since even if hybrid LDOS peaks are high, their integrated signature is small. While multiple-emitter decay traces are difficult to interpret due to large variations in lifetime (i.e., in distance to the antenna), these single-emitter studies allow an unambiguous lifetime measurement. Combined with the hybrid boost, this measures the absolute LDOS enhancement at the hybrid peak.

The single quantum dot on a hybrid with a short $(88 \mathrm{~nm})$ antenna shows an emission lifetime of 25.9 ns, a modest decrease by a factor $\sim 6$ compared to quantum dots on a flat glass substrate (which have an average lifetime of $153 \mathrm{~ns}$ ). The hybrid boost, however, is significant (4.9), in agreement with the multi-quantum-dot results of Figure 3. This brings the total measured hybrid LDOS increase, which is the product of boost (4.9) and bare antenna LDOS (6), to $\sim 29 \times$. The quantum dot on a hybrid with a longer $(128 \mathrm{~nm})$, resonant antenna, in contrast, shows a stronger lifetime decrease of 153/8.5 $=18 \times$, yet a weak hybrid enhancement factor (1.2), bringing the total LDOS increase to $\sim 22 \times$, as the system is in the regime of induced transparency. Results for other structures are shown in the Supporting Information. These results show unambiguously that single quantum emitters can be coupled to hybrid plasmonic-photonic modes and experience the strong LDOS variations offered by them.

\section{CONCLUSIONS}

To conclude, we reported antenna-cavity hybrid modes with quality factors on the order of $10^{3}$, yet distinct plasmonic confinement signatures in LDOS, as probed by single quantum emitter spectra. The hybrid LDOS arises from a coherent interplay between cavity and antenna contributions, as evident from the fact that emission spectra can be shaped into any Fano lineshape from pure suppression to near-Lorentzian enhancement. For cavities red-detuned from the antenna resonance, LDOS can be significantly enhanced with respect to that of the antenna alone, with measured "hybrid boost" factors up to $\sim 14$. Our single quantum emitter studies furthermore evidence total LDOS enhancements of up to 29, boosted 5fold over that due to the bare plasmon antenna. These results are made possible largely by the chemically enhanced lithographic technique developed in this work, which allows precise placement of single quantum dots inside hybrid resonators.

This work demonstrates the potential of hybrid systems as a powerful platform for light-matter interaction that can be extended to significantly outperform the metrics reported here. While the hybrids in this work did not outperform the bare cavities (estimated Purcell factors of $\sim 108$ for the $4 \mu \mathrm{m}$ disks, based on simulations) because the antennas provided insufficient confinement, this was proposed to be possible with improved antennas. ${ }^{18,43}$ Hybrids improve in proportion to bare cavity Purcell factor and bare antenna enhancement factor. Both can be improved by orders of magnitude compared to the numbers that we report for our hybrids, by turning to photonic crystal microcavities (mode volumes $10^{2}-$ $10^{3}$ smaller than those of our disks) and gap antennas (LDOS a factor of $10^{2}-10^{4}$ in excess of that at nanorods). This gives room to optimally match the hybrid $Q$ and Purcell factor to diverse emitters. Notably, we envision strong light-matter interaction with single emitters under conditions that bridge the gap between plasmonic picocavities and cavity-QED in III-V photonic crystal systems ${ }^{44}$ and furthermore predict an important role for hybrids in applications that benefit from high LDOS at a localized hot spot and high $Q^{43}$ such as sensing, spectroscopy, optomechanics, lasers, and nano-LEDs.

The data sets generated and/or analyzed during the current study are available from the corresponding author on reasonable request.

\section{METHODS}

Fabrication of Hybrid Cavity-Antenna Systems. The hybrid systems studied in this work are composed of silicon nitride microdisks (4-15 $\mu \mathrm{m}$ in diameter), dressed with a single aluminum nanoantenna. Microdisks are etched into a $200 \mathrm{~nm}$ layer of low-loss stoichiometric silicon nitride $\left(\mathrm{Si}_{3} \mathrm{~N}_{4}\right)$ on silicon using an inductively coupled plasma (ICP) reactive ion etching (RIE). We etch for $100 \mathrm{~s}$ using a mix of $\mathrm{SF}_{6}$ and $\mathrm{CHF}_{3}$ gases at flow rates of 16 and 80 standard cubic centimeters per minute $(\mathrm{sccm})$, respectively, with $50 \mathrm{~W}$ RIE forward power and $500 \mathrm{~W}$ ICP power at a gas pressure of $9 \mathrm{mTorr}$ and a temperature of $0{ }^{\circ} \mathrm{C}$. The resist mask is composed of a $450 \mathrm{~nm}$ layer of positive resist (CSAR 6200, Allresist $\mathrm{GmbH}$ ), exposed in an e-beam lithography system (Raith Voyager, $50 \mathrm{kV}$ ) using an electron dose of $160 \mu \mathrm{C} / \mathrm{cm}^{2}$ and developed in pentyl acetate $(120 \mathrm{~s})$ and $o$ xylene $(7 \mathrm{~s})$. After the plasma etch, samples are cleaned and immediately underetched in a $40 \mathrm{wt} \%$ potassium hydroxide $(\mathrm{KOH})$ solution at $70{ }^{\circ} \mathrm{C}$, which etches the underlying silicon to create pillars with roughly pyramidal shapes. Etch times are around $4 \mathrm{~min}$.

To position the antenna, we perform a second e-beam lithography step. We spin-coat a layer of methyl methacrylate (MMA, Microchem MMA 8.5, dissolved in ethyl lactate), followed by a layer of poly(methyl methacrylate) (PMMA, Microchem 950 PMMA, dissolved in anisole). The layer thicknesses at the antenna location near the disk edge, as calibrated using focused-ion-beam milling on similar samples, are $150 \mathrm{~nm}$ (MMA) and $60 \mathrm{~nm}$ (PMMA). During the e-beam exposure $\left(500 \mu \mathrm{C} / \mathrm{cm}^{2}, 50 \mathrm{kV}\right)$, we use markers that were defined in the same step as the disks to align antennas to the disks. We write rectangular antennas of $50 \mathrm{~nm}$ width and different lengths between 60 and $180 \mathrm{~nm}$, positioned $300 \mathrm{~nm}$ from the disk edge, with the antenna long axis always aligned in the radial direction. The sample is then developed in a 1:3 mixture of MIBK and IPA (80 s), and a $40 \mathrm{~nm}$ layer of aluminum is deposited using thermal evaporation at a rate of $0.05 \mathrm{~nm} / \mathrm{s}$ and a pressure of $5 \times 10^{-7}$ mbar. Finally we lift off the excess metal in a warm acetone bath (50 ${ }^{\circ} \mathrm{C}$ ) for $\sim 30 \mathrm{~min}$.

The samples without quantum dots, which are studied with taperfiber spectroscopy, are subsequently covered in a several- $\mu \mathrm{m}$-thick layer of positive UV resist (MICROPOSIT S1800) for protection, 
after which the sample height is reduced by $\sim 150 \mu \mathrm{m}$ using a diamond saw, leaving only a thin mesa supporting the microdisks. After sawing, the resist is removed in a $45{ }^{\circ} \mathrm{C}$ acetone bath.

Further fabrication details can be found in the Supporting Information.

Selective Positioning of Quantum Dots in Hybrid Systems. To position quantum dots (Invitrogen Qdot 800 ITK Organic, Q21771MP) at the antenna tips, we use a method that is similar to that developed by Curto et al. ${ }^{35,36}$ for binding the same quantum dots to gold nanoantennas. The main difference is the linker chemistry: we use a functional group that can bind specifically to aluminum rather than to gold. Moreover, we use a thiol to bind directly to the QD, rather than binding to the QD ligands. Starting from samples containing cavity-antenna hybrids, we first spin-coat a $1100 \mathrm{~nm}$ layer of PMMA, which we measured to be $500 \mathrm{~nm}$ thick at the antenna location on the disks. Following the same procedure for alignment, ebeam exposure, and development as used for writing the antennas, we define holes of 60 or $120 \mathrm{~nm}$ diameter, centered at the antenna apex pointing to the disk center. For the multiple-quantum-dot measurements shown in Figure 3, we used $120 \mathrm{~nm}$ holes, while for the singlequantum-dot measurements shown in Figure 4, we use both 120 and $60 \mathrm{~nm}$ holes.

To increase the selectivity of quantum dot binding to the aluminum antennas rather than the SiN disk surface, we functionalize the exposed aluminum surfaces using a self-assembled monolayer of 12-mercaptododecylphosphonic acid (MDPA, purchased from SigmaAldrich, 95\% purity) following a recipe by Attavar et al. ${ }^{45}$ We then proceed with the quantum dot binding. Samples are immersed in a 10 $\mathrm{nM}$ solution of Qdot 800 quantum dots in decane for $10 \mathrm{~min}$, subsequently rinsed in pure decane $(1 \mathrm{~min})$, and then kept in toluene for $\sim 1 \mathrm{~h}$, after which they are dried and are ready for use. Note how this recipe differs from that of Curto et al..$^{35}$ (which also used different linker molecules, ligands, and solvents) in the immersion time of 10 min, rather than $24 \mathrm{~h}$. We found that longer immersion times lead to quantum dots present also outside the exposed areas, which is likely due to diffusion through the PMMA layer. ${ }^{46}$ At a $24 \mathrm{~h}$ immersion time, the sample is fully covered with quantum dots with barely any difference between areas with and without PMMA.

Our recipe typically results in quantum dots being positioned with excellent accuracy and large selectivity, i.e., no quantum dots in the areas covered in PMMA. For more details and information about the quantum dots, see the Supporting Information.

Processing Fiber-Coupled Spectra. To obtain the line widths and frequency shifts shown in Figure $2 \mathrm{j}, \mathrm{k}$, we record and fit fiber transmission, reflection, and scattering spectra as follows. For each hybrid structure, we measure a broadband (several $\mathrm{nm}$ range) spectrum by sweeping the laser wavelength and recording a time trace of the diode signals. Time traces are converted to spectra using the wavelength output signal from the laser, which is recorded simultaneously and corresponds directly to the laser wavelength. We find the modes that do not show scattering off the edge of the silicon pedestal, which correspond to the higher-order radial modes ( $m \leq 2$ in our case). Comparison of the frequency spacings between modes to those obtained from simulations then allows identification of the $m_{r}=0$ and $m_{r}=1$ modes. We subsequently record a narrowband spectrum of each mode by fine-tuning the laser wavelength in a small range (approximately $\pm 40 \mathrm{GHz}$ ) using a built-in piezoelectric actuator. For these scans, the frequency axis is calibrated by simultaneously recording a transmission spectrum of a reference cavity (Thorlabs SA201-5B Fabry-Perot cavity) with a known free-spectral range of $10 \mathrm{GHz}$. As this scan is done step-bystep, we can also record a camera image at each frequency point. From the camera data, we integrate the pixel counts from a small region around the antenna to obtain an additional low-noise scattering spectrum.

We fit the obtained spectra using a global least-squares fit to the transmission, reflection, and scattering data, using expressions from coupled-mode theory (see Supporting Information). We separately fit both the narrowband piezo scan data and a narrowband region of the broadband scan data. For the piezo scans, we use the integrated camera counts as scattering data, while for the broadband scans we use the photodiode trace. Although usually there is good agreement between the two scan methods, occasionally there are line width differences of up to $\sim 30 \%$, which we attribute to a slightly nonlinear camera response in the low-intensity regime. To avoid thermal bistability effects, we always do scans with increasing and decreasing wavelength and verify that the obtained line widths are similar (typically within $10 \%$ ).

We measure cavities of 15,12 , and $8 \mu \mathrm{m}$ diameter. For each diameter, there are cavities with 10 different antenna lengths between 68 and $188 \mathrm{~nm}$ and a thickness of $40 \mathrm{~nm}$. For each combination of diameter and antenna length, there are two cavities, bringing the total number of measured cavities to 60 . On each cavity, both the $m_{r}=0$ and the $m_{r}=1$ modes are measured. We average the results from narrowband and broadband scans and from scans with increasing and decreasing wavelength. Cavities with visible dirt and spectra with a failed fit routine or insufficient signal-to-noise ratio are excluded from the analysis. For hybrids with line widths similar to or larger than the maximum piezoscan range of $\sim 80 \mathrm{GHz}$, we use only the results from the broadband scans.

Predicting Shifts and Broadenings Using Cavity Perturbation Theory and FEM Simulations. To compare our experimentally obtained broadenings and frequency shifts (shown in Figure $2(\mathrm{j}, \mathrm{k}))$ to cavity perturbation theory (eq 1 ), we require knowledge of the antenna polarizability $\alpha$ and the cavity effective mode volume $V_{\text {eff }}$ Both are retrieved from a finite element simulation (COMSOL multiphysics, v5.1) of the antenna or cavity separately.

The cavity eigenmodes are found in a $2 \mathrm{D}$ simulation (using the axial symmetry), and effective mode volume is calculated as

$$
V_{\text {eff }}=\frac{\int \mathrm{d} \boldsymbol{r} \epsilon(\boldsymbol{r})\left|\mathbf{E}_{\mathrm{c}}(\boldsymbol{r})\right|^{2}}{\epsilon\left(\boldsymbol{r}_{\mathrm{a}}\right)\left|\hat{\mathbf{p}} \cdot \mathbf{E}_{\mathrm{c}}\left(\boldsymbol{r}_{\mathrm{a}}\right)\right|^{2}}
$$

i.e., using the cavity field $\mathrm{E}_{\mathrm{c}}$ at the antenna location $\boldsymbol{r}_{\mathrm{a}}$ contrary to the usual definition of a cavity mode volume $\mathrm{e}^{47,48}$ that uses the maximum field in the cavity mode instead. Here, $\hat{\mathbf{p}}$ is the direction of the antenna dipole moment, taken along the cavity radial direction. Values for $V_{\text {eff }}$ are shown in Table 1 in the Supporting Information. Based on SEM images of the disks, we assume the 15 and $12 \mu \mathrm{m}$ (8 $\mu \mathrm{m})$ disks to have $50 \mathrm{~nm}(100 \mathrm{~nm})$ smaller radii than designed for. Antennas, which were designed to be centered $300 \mathrm{~nm}$ from the disk edge, are therefore assumed to be $250 \mathrm{~nm}(200 \mathrm{~nm})$ from the edge.

To obtain $\alpha$, we perform simulations of aluminum nanorod antennas on an infinite $\mathrm{Si}_{3} \mathrm{~N}_{4}$ substrate. Antenna width and thickness are taken as 58 and $40 \mathrm{~nm}$, respectively, and we assume a tapered shape in the vertical direction with a taper angle of $60^{\circ}$, based on cross-cut images of other evaporated structures. The aluminum refractive index is taken from tabulated data, ${ }^{49}$ and we include a $3 \mathrm{~nm}$ alumina shell. We drive the antenna with a plane wave at normal incidence from the air side, polarized along the antenna long axes ( $x$ axis) and calculate scattered and absorbed power as well as induced dipole moments (along all three axes) as a function of frequency. Division of the dipole moments by the incident field at the antenna location yields antenna polarizability tensor elements $\alpha_{x x}, \alpha_{x y}$ and $\alpha_{x z}$ We find that $\alpha_{x x}$ dominates the polarizability for all antenna sizes at our wavelength of interest, so we ignore other tensor elements. For calculating perturbations, polarizability is evaluated at the cavity resonance frequency (taken to be fixed at $387 \mathrm{THz}$ ).

Fluorescence Measurements: Experimental methods. Fluorescence spectra and time-correlated single-photon counting (TCSPC) measurements were performed in a fluorescence microscope, which is an adapted version of the setup reported in our earlier work $^{50}$ and is shown in the Supporting Information. The sample is illuminated through an objective (Olympus MPlan IR, 100X, NA 0.95 ) by a pulsed pump laser (640 nm, PicoQuant LDH-P-C-640B pulsed diode laser, 500 ps pulse width). Unless stated otherwise, measurements were done with the pump beam focused on the sample. In the detection path, this pump is removed by a dichroic beamsplitter and a long-pass filter. Pump and detection polarization are controlled by linear polarizers. Fluorescence is collected either on 
a camera, a fiber-coupled spectrometer (Andor Shamrock 303i, equipped with an iVac DR316B-LDC-DD detector), or two avalanche photodiodes (APDs) in a Hanbury-Brown-Twiss configuration. ${ }^{51}$ The spectrometer uses a multimode fiber of $10 \mu \mathrm{m}$ core size, which translates to a detection area of $\sim 1 \mu \mathrm{m}$ on the sample. We performed broadband $(\Delta \lambda=314 \mathrm{~nm}$, resolution $\sim 0.4 \mathrm{~nm}=0.2 \mathrm{THz}$ at $780 \mathrm{~nm}$ wavelength $)$ or high-resolution $(\Delta \lambda=62 \mathrm{~nm}$, resolution $\sim 0.1 \mathrm{~nm}=$ $0.05 \mathrm{THz}$ ) measurements using a 300 or 1200 lines $/ \mathrm{mm}$ spectrometer grating, respectively. Photon arrival times from the APDs (Excelitas SPCM-AQRH-14-FC single photon counting modules, 350 ps timing resolution) are used to measure excited state decay curves and the second-order correlation function $g^{(2)}$. APD counts and pump pulse events are recorded on a Becker \& Hickl DPC 230 timing card.

To avoid background signal from intrinsic silicon nitride fluorescence, ${ }^{52}$ we use a pump laser of $640 \mathrm{~nm}$ wavelength with repetition rate variable between 2.5 and $40 \mathrm{MHz}$. We found that when using a $532 \mathrm{~nm}$ pump laser instead, intrinsic fluorescence from the $\mathrm{Si}_{3} \mathrm{~N}_{4}$ was clearly visible and of similar intensity to the quantum dot fluorescence (although peaked at a shorter wavelength around 650 $\mathrm{nm})$. With the $640 \mathrm{~nm}$ laser, in contrast, fluorescence from the quantum dots is far dominant.

All multi-quantum-dot spectra (as used in Figure 3) are acquired using a 60 or $120 \mathrm{~s}$ camera integration time, with the laser set to a 20 $\mathrm{MHz}$ repetition rate. For single quantum dot spectra, integration times were between 60 and $240 \mathrm{~s}$, at the same laser repetition rate. Pump pulse energy (at the sample) was set to $40-140 \mathrm{fJ} / \mathrm{pulse}$, corresponding to a pump fluence of 40 to $120 \mu \mathrm{J} / \mathrm{cm}^{2} /$ pulse. At much higher pump fluence $\left(>200 \mu \mathrm{J} / \mathrm{cm}^{2} /\right.$ pulse $)$, the background contribution from silicon nitride and aluminum fluorescence becomes more prominent. Background spectra, taken without pump light, are subtracted. Pixels with anomalously high counts (usually attributed to cosmic rays) are removed in postprocessing. The high-resolution spectra may show intensity fringes due to an etalon effect in the camera chip itself, with amplitudes up to $50 \%$ of the signal. As they occur at a specific frequency, we remove them by suppressing the corresponding frequency components in a Fourier transform of the signal.

Measurements with the APDs were done with the laser set to 10 $\mathrm{MHz}$ repetition rate, acquisition times of $30-120 \mathrm{~s}(60-300 \mathrm{~s})$ for multi-quantum-dot (single-quantum-dot) measurements, and individual APD count rates around 5-50 kHz.

Postselection of the Single-Quantum-Dot Bright States. The single quantum dots in our hybrids often spend most time during our measurements in the dark state. This decreases the signal-tobackground ratio in our photon correlation measurements. In our case, the background signal is composed of fluorescence and Raman scattering from the SiN and fluorescence from the aluminum antenna. To improve the signal-to-background ratio, we perform a postselection of data, selecting only the data points from times that the quantum dot was in the bright state. This procedure is demonstrated in the Supporting Information for a single quantum dot on a flat $\mathrm{SiN}$ substrate $(200 \mathrm{~nm} \mathrm{SiN}$ on $\mathrm{Si}$ ). We first bin the APD counts into 20 $\mathrm{ms}$ time bins and show an intensity time trace. We then fit a Gaussian to the histogram of this data to find the dark state count rate. We then pick a threshold value and split our data into "ON" and "OFF", depending on whether the total count rate in that $20 \mathrm{~ms}$ bin was above or below the threshold. Finally, we perform the usual correlations on the $\mathrm{ON}$ and OFF data separately to calculate fluorescence decay traces and $g^{(2)}$. Results are shown in the Supporting Information, both for the quantum dot on a flat substrate and for quantum dots in a hybrid structure. These demonstrate "purification" of the quantum dot decay curves, which go from nonexponential to a clear biexponential decay in the ON-state data, and of $g^{(2)}$, which shows much more clear antibunching in the ONstate data.

\section{ASSOCIATED CONTENT}

\section{Supporting Information}

The Supporting Information is available free of charge at https://pubs.acs.org/doi/10.1021/acsnano.0c05233.

Further details about sample fabrication, measurement procedure, and data processing, as well as supporting data $(\mathrm{PDF})$

\section{AUTHOR INFORMATION}

\section{Corresponding Author}

A. Femius Koenderink - Center for Nanophotonics, AMOLF, 1098 XG Amsterdam, Netherlands; Van der Waals-Zeeman Instituut, Institute of Physics, Universiteit van Amsterdam, 1090 GL Amsterdam, Netherlands; () orcid.org/0000-0003-16175748; Email: f.koenderink@amolf.nl

\section{Authors}

Hugo M. Doeleman - Van der Waals-Zeeman Instituut, Institute of Physics, Universiteit van Amsterdam, 1090 GL Amsterdam, Netherlands; Center for Nanophotonics, AMOLF, 1098 XG Amsterdam, Netherlands; 이이.org/0000-00032976-8294

Christian D. Dieleman - Center for Nanophotonics, AMOLF, 1098 XG Amsterdam, Netherlands

Christiaan Mennes - Center for Nanophotonics, AMOLF, 1098 XG Amsterdam, Netherlands

Bruno Ehrler - Center for Nanophotonics, AMOLF, 1098 XG Amsterdam, Netherlands; 이이. orcid.org/0000-0002-5307-3241

Complete contact information is available at:

https://pubs.acs.org/10.1021/acsnano.0c05233

\section{Notes}

The authors declare no competing financial interest.

\section{ACKNOWLEDGMENTS}

This work is part of the research program Hybrid nanophotonic architectures for ultrafast quantum optics [NWO-Vici] with project number 680.47 .621 , which is financed by the Dutch Research Council (NWO), and was performed at the research institute AMOLF. The authors are grateful to the Amsterdam Nanocenter staff for their support regarding nanofabrication.

\section{REFERENCES}

(1) Noda, S.; Fujita, M.; Asano, T. Spontaneous-Emission Control by Photonic Crystals and Nanocavities. Nat. Photonics 2007, 1, 449458 .

(2) Lodahl, P.; Mahmoodian, S.; Stobbe, S. Interfacing Single Photons and Single Quantum Dots with Photonic Nanostructures. Rev. Mod. Phys. 2015, 87, 347-400.

(3) Koenderink, A. F. Single-Photon Nanoantennas. ACS Photonics 2017, 4, 710-722.

(4) Altug, H.; Englund, D.; Vučković, J. Ultrafast Photonic Crystal Nanocavity Laser. Nat. Phys. 2006, 2, 484-488.

(5) Oulton, R. F.; Sorger, V. J.; Zentgraf, T.; Ma, R. M.; Gladden, C.; Dai, L.; Bartal, G.; Zhang, X. Plasmon Lasers at Deep Subwavelength Scale. Nature 2009, 461, 629-632.

(6) Vollmer, F.; Arnold, S. Whispering-Gallery-Mode Biosensing: Label-Free Detection Down to Single Molecules. Nat. Methods 2008, 5, 591-596.

(7) Benz, F.; Schmidt, M. K.; Dreismann, A.; Chikkaraddy, R.; Zhang, Y.; Demetriadou, A.; Carnegie, C.; Ohadi, H.; de Nijs, B.; Esteban, R.; Aizpurua, J.; Baumberg, J. J. Single-Molecule Optomechanics in "Picocavities". Science 2016, 354, 726-729. 
(8) Maser, A.; Gmeiner, B.; Utikal, T.; Götzinger, S.; Sandoghdar, V. Few-Photon Coherent Nonlinear Optics with a Single Molecule. Nat. Photonics 2016, 10, 450-453.

(9) Aspelmeyer, M.; Kippenberg, T. J.; Marquardt, F. Cavity Optomechanics. Rev. Mod. Phys. 2014, 86, 1391-1452.

(10) Schuller, J. A.; Barnard, E. S.; Cai, W.; Jun, Y. C.; White, J. S.; Brongersma, M. L. Plasmonics for Extreme Light Concentration and Manipulation. Nat. Mater. 2010, 9, 193-204.

(11) Hoang, T. B.; Akselrod, G. M.; Argyropoulos, C.; Huang, J.; Smith, D. R.; Mikkelsen, M. H. Ultrafast Spontaneous Emission Source Using Plasmonic Nanoantennas. Nat. Commun. 2015, 6, 7788.

(12) Hu, S.; Khater, M.; Salas-Montiel, R.; Kratschmer, E.; Engelmann, S.; Green, W. M. J.; Weiss, S. M. Experimental Realization of Deep-Subwavelength Confinement in Dielectric Optical Resonators. Sci. Adv. 2018, 4, No. eaat2355.

(13) Foreman, M. R.; Vollmer, F. Level Repulsion in Hybrid Photonic-Plasmonic Microresonators for Enhanced Biodetection. Phys. Rev. A: At., Mol., Opt. Phys. 2013, 88, 23831.

(14) Zhao, X.-M.; Xia, Y.; Whitesides, G. M. Soft Lithographic Methods for Nano-Fabrication. J. Mater. Chem. 1997, 7, 1069-1074.

(15) Thakkar, N.; Rea, M. T.; Smith, K. C.; Heylman, K. D.; Quillin, S. C.; Knapper, K. A.; Horak, E. H.; Masiello, D. J.; Goldsmith, R. H. Sculpting Fano Resonances to Control Photonic-Plasmonic Hybridization. Nano Lett. 2017, 17, 6927-6934.

(16) Pan, F.; Smith, K. C.; Nguyen, H. L.; Knapper, K. A.; Masiello, D. J.; Goldsmith, R. H. Elucidating Energy Pathways through Simultaneous Measurement of Absorption and Transmission in a Coupled Plasmonic-Photonic Cavity. Nano Lett. 2020, 20, 50-58.

(17) Frimmer, M.; Koenderink, A. F. Superemitters in Hybrid Photonic Systems: A Simple Lumping Rule for the Local Density of Optical States and Its Breakdown at the Unitary Limit. Phys. Rev. B: Condens. Matter Mater. Phys. 2012, 86, 235428.

(18) Doeleman, H. M.; Verhagen, E.; Koenderink, A. F. AntennaCavity Hybrids: Matching Polar Opposites for Purcell Enhancements at Any Linewidth. ACS Photonics 2016, 3, 1943-1951.

(19) Dezfouli, M. K.; Gordon, R.; Hughes, S. Modal Theory of Modified Spontaneous Emission of a Quantum Emitter in a Hybrid Plasmonic Photonic-Crystal Cavity System. Phys. Rev. A: At., Mol., Opt. Phys. 2017, 95, 013846.

(20) Barth, M.; Schietinger, S.; Fischer, S.; Becker, J.; Nüsse, N.; Aichele, T.; Löchel, B.; Sönnichsen, C.; Benson, O. Nanoassembled Plasmonic-Photonic Hybrid Cavity for Tailored Light-Matter Coupling. Nano Lett. 2010, 10, 891-895.

(21) Zhang, T.; Callard, S.; Jamois, C.; Chevalier, C.; Feng, D.; Belarouci, A. Plasmonic-Photonic Crystal Coupled Nanolaser. Nanotechnology 2014, 25, 315201.

(22) Baaske, M. D.; Vollmer, F. Optical Observation of Single Atomic Ions Interacting with Plasmonic Nanorods in Aqueous Solution. Nat. Photonics 2016, 10, 733-739.

(23) Chen, B.; Bruck, R.; Traviss, D.; Khokhar, A. Z.; Reynolds, S.; Thomson, D. J.; Mashanovich, G. Z.; Reed, G. T.; Muskens, O. L. Hybrid Photon-Plasmon Coupling and Ultrafast Control of Nanoantennas on a Silicon Photonic Chip. Nano Lett. 2018, 18, 610-617.

(24) Ruesink, F.; Doeleman, H. M.; Verhagen, E.; Koenderink, A. F. Controlling Nanoantenna Polarizability through Backaction via a Single Cavity Mode. Phys. Rev. Lett. 2018, 120, 206101.

(25) Cognée, K. G.; Doeleman, H. M.; Lalanne, P.; Koenderink, A. F. Cooperative Interactions between Nano-Antennas in a High-Q Cavity for Unidirectional Light Sources. Light: Sci. Appl. 2019, 8, 115.

(26) Caselli, N.; Intonti, F.; La China, F.; Biccari, F.; Riboli, F.; Gerardino, A.; Li, L.; Linfield, E. H.; Pagliano, F.; Fiore, A.; Gurioli, M. Generalized Fano Lineshapes Reveal Exceptional Points in Photonic Molecules. Nat. Commun. 2018, 9, 396.

(27) Kippenberg, T. J. A. Nonlinear Optics in Ultra-High $Q$ Whispering-Gallery Optical Microcavities. Ph.D. thesis; California Institute of Technology, 2004.

(28) Hauer, B. D.; Kim, P. H.; Doolin, C.; MacDonald, A. J.; Ramp, H.; Davis, J. P. On-Chip Cavity Optomechanical Coupling. EPJ. Techn. Instrum. 2014, 1, 4.
(29) Bethe, H.; Schwinger, J. Perturbation Theory for Cavities; Massachusetts Institute of Technology, Radiation Laboratory: Cambridge, MA, 1943.

(30) Borselli, M. High-Q Microresonators as Lasing Elements for Silicon Photonics. Ph.D. thesis; California Institute of Technology, 2006.

(31) Lalouat, L.; Cluzel, B.; Velha, P.; Picard, E.; Peyrade, D.; Hugonin, J. P.; Lalanne, P.; Hadji, E.; de Fornel, F. Near-Field Interactions Between a Subwavelength Tip and a Small-Volume Photonic-Crystal Nanocavity. Phys. Rev. B: Condens. Matter Mater. Phys. 2007, 76, 041102.

(32) Mujumdar, S.; Koenderink, A. F.; Sünner, T.; Buchler, B. C.; Kamp, M.; Forchel, A.; Sandoghdar, V. Near-Field Imaging and Frequency Tuning of a High-Q Photonic Crystal Membrane Microcavity. Opt. Express 2007, 15, 17214.

(33) Burresi, M.; Kampfrath, T.; van Oosten, D.; Prangsma, J. C.; Song, B. S.; Noda, S.; Kuipers, L. Magnetic Light-Matter Interactions in a Photonic Crystal Nanocavity. Phys. Rev. Lett. 2010, 105, 123901.

(34) Cognée, K. G.; Yan, W.; La China, F.; Balestri, D.; Intonti, F.; Gurioli, M.; Koenderink, A. F.; Lalanne, P. Mapping Complex Mode Volumes with Cavity Perturbation Theory. Optica 2019, 6, 269.

(35) Curto, A. G.; Volpe, G.; Taminiau, T. H.; Kreuzer, M. P.; Quidant, R.; van Hulst, N. F. Unidirectional Emission of a Quantum Dot Coupled to a Nanoantenna. Science 2010, 329, 930-933.

(36) Ureña, E. B.; Kreuzer, M. P.; Itzhakov, S.; Rigneault, H.; Quidant, R.; Oron, D.; Wenger, J. Excitation Enhancement of a Quantum Dot Coupled to a Plasmonic Antenna. Adv. Mater. 2012, 24, OP314-OP320.

(37) Novotny, L.; Hecht, B. Principles of Nano-Optics, 2nd ed.; Cambridge University Press: New York, 2012.

(38) Chizhik, A.; Schleifenbaum, F.; Gutbrod, R.; Chizhik, A.; Khoptyar, D.; Meixner, A. J.; Enderlein, J. Tuning the Fluorescence Emission Spectra of a Single Molecule with a Variable Optical Subwavelength Metal Microcavity. Phys. Rev. Lett. 2009, 102, 073002.

(39) Toninelli, C.; Delley, Y.; Stöferle, T.; Renn, A.; Götzinger, S.; Sandoghdar, V. A Scanning Microcavity for In Situ Control of SingleMolecule Emission. Appl. Phys. Lett. 2010, 97, 021107.

(40) Kaupp, H.; Deutsch, C.; Chang, H.-C.; Reichel, J.; Hänsch, T. W.; Hunger, D. Scaling Laws of the Cavity Enhancement for Nitrogen-Vacancy Centers in Diamond. Phys. Rev. A: At., Mol., Opt. Phys. 2013, 88, 053812.

(41) Rabouw, F. T.; Prins, P. T.; Norris, D. J. Europium-Doped NaYF4 Nanocrystals as Probes for the Electric and Magnetic Local Density of Optical States Throughout the Visible Spectral Range. Nano Lett. 2016, 16, 7254-7260.

(42) Pellegrino, D.; Balestri, D.; Granchi, N.; Ciardi, M.; Intonti, F.; Pagliano, F.; Silov, A. Y.; Otten, F. W.; Wu, T.; Vynck, K.; Lalanne, P.; Fiore, A.; Gurioli, M. Non-Lorentzian Local Density of States in Coupled Photonic Crystal Cavities Probed by Near- and Far-Field Emission. Phys. Rev. Lett. 2020, 124, 123902.

(43) Palstra, I. M.; Doeleman, H. M.; Koenderink, A. F. Hybrid Cavity-Antenna Systems for Quantum Optics Outside the Cryostat? Nanophotonics 2019, 8, 1513-1531.

(44) Gurlek, B.; Sandoghdar, V.; Martín-Cano, D. Manipulation of Quenching in Nanoantenna-Emitter Systems Enabled by External Detuned Cavities: A Path to Enhance Strong-Coupling. ACS Photonics 2018, 5, 456-461.

(45) Attavar, S.; Diwekar, M.; Linford, M. R.; Davis, M. A.; Blair, S. Passivation of Aluminum with Alkyl Phosphonic Acids for Biochip Applications. Appl. Surf. Sci. 2010, 256, 7146-7150.

(46) Rabouw, F. T.; Frimmer, M.; Mohtashami, A.; Koenderink, A. F. Nanoscale Lithographic Positioning of Fluorescing Quantum Dot Nanocrystals on Planar Samples. Opt. Mater. 2013, 35, 1342-1347.

(47) Purcell, E. M. Spontaneous Emission Probabilities at Radio Frequencies. Phys. Rev. 1946, 69, 681.

(48) Kippenberg, T. J.; Spillane, S. M.; Vahala, K. J. Demonstration of Ultra-High-Q Small Mode Volume Toroid Microcavities on a Chip. Appl. Phys. Lett. 2004, 85, 6113-6115. 
(49) Rakić, A. D.; Djurišíc, A. B.; Elazar, J. M.; Majewski, M. L. Optical Properties of Metallic Films for Vertical-Cavity Optoelectronic Devices. Appl. Opt. 1998, 37, 5271.

(50) Frimmer, M.; Chen, Y.; Koenderink, A. F. Scanning Emitter Lifetime Imaging Microscopy for Spontaneous Emission Control. Phys. Rev. Lett. 2011, 107, 123602.

(51) Loudon, R. The Quantum Theory of Light; Oxford Science Publications: Oxford, 1983.

(52) Barth, M.; Nüsse, N.; Stingl, J.; Löchel, B.; Benson, O. Emission Properties of High-Q Silicon Nitride Photonic Crystal Heterostructure Cavities. Appl. Phys. Lett. 2008, 93, 021112. 